\title{
On Some Fractional Order Hardy Inequalities
}

\author{
HANS P. HEINIG ${ }^{a}$, ALOIS KUFNER $^{b}$ and LARS-ERIK PERSSON ${ }^{c}$ \\ ${ }^{a}$ Department of Mathematics \& Statistics, McMaster University, \\ Hamilton, Ontario, L8S 4K1, Canada \\ ${ }^{b}$ Mathematical Institute, Czech Academy of Sciences, \\ Žitná 25, 11567 Praha 1, Czech Republic \\ ${ }^{c}$ Department of Mathematics, Luleå University, \\ S-971 87 Luleå, Sweden
}

(Received 6 May 1996)

Weighted inequalities for fractional derivatives (= fractional order Hardy-type inequalities) have recently been proved in [4] and [1]. In this paper, new inequalities of this type are proved and applied. In particular, the general mixed norm case and a general twodimensional weight are considered. Moreover, an Orlicz norm version and a multidimensional fractional order Hardy inequality are proved. The connections to related results are pointed out.

Keywords: Hardy inequality; weight function; fractional order derivative.

AMS Subject Classification: 26D15, 46E30

\section{INTRODUCTION}

Using the notation $\|f\|_{s, w}$ for the norm in the weighted Lebesgue space $L^{s}(0, \infty ; w)=L^{s}(w)$,

$$
\|f\|_{s, w}=\left(\int_{0}^{\infty}|f(t)|^{s} w(t) d t\right)^{1 / s}
$$

with $1<s<\infty$ and $w=w(t)$ a weight function on $(0, \infty)$, the Hardy inequality can be expressed in the form

$$
\|u\|_{q, w_{0}} \leq C\left\|u^{\prime}\right\|_{p, w_{1}}
$$


with a constant $C>0$ independent of $u$, i.e., under certain conditions, the weighted $L^{q}$-norm of the function $u$ can be estimated by a suitable weighted $L^{p}$-norm of its (first order) derivative $u^{\prime}$.

The natural question arises whether it is possible to extend inequality (1.1) to "fractional order derivatives" $u^{(\lambda)}$ with $0<\lambda<1$, i.e., under what conditions we can derive inequalities of the type

$$
\|u\|_{q, w_{0}} \leq C\left\|u^{(\lambda)}\right\|_{p, w_{\lambda}}
$$

and/or

$$
\left\|u^{(\lambda)}\right\|_{q, w_{\lambda}} \leq C\left\|u^{\prime}\right\|_{p, w_{1}} .
$$

The first problem which arises is how to understand the expression $\left\|u^{(\lambda)}\right\|_{q, w}$. Let us mention that for the "non-weighted" case (i.e., for $w(t) \equiv 1)$ the following definition of $\left\|u^{(\lambda)}\right\|_{s, 1}=\left\|u^{(\lambda)}\right\|_{s}$ is commonly used:

$$
\left\|u^{(\lambda)}\right\|_{s}=\left(\int_{0}^{\infty} \int_{0}^{\infty} \frac{|u(x)-u(y)|^{s}}{|x-y|^{1+\lambda s}} d x d y\right)^{1 / s}, 1<s<\infty, 0<\lambda<1 .
$$

For this special case, the following inequality holds:

$$
\int_{0}^{\infty}|u(x)|^{p} x^{-\lambda p} d x \leq C^{p} \int_{0}^{\infty} \int_{0}^{\infty} \frac{|u(x)-u(y)|^{p}}{|x-y|^{1+\lambda p}} d x d y
$$

which is inequality (1.2) for the special case

$$
p=q, w_{\lambda}(x) \equiv 1, w_{0}(x)=x^{-\lambda p} .
$$

Inequality (1.5) was derived by Grisvard [2] provided

$$
1<p<\infty, \lambda \neq \frac{1}{p}, u \in C_{0}^{\infty}(0, \infty) .
$$

In fact, he rediscovered an earlier result of Jakovlev [3] who has shown that

$$
\int_{0}^{\infty}|u(x)-u(0)|^{p} x^{-\lambda p} d x \leq C^{p} \int_{0}^{\infty} \int_{0}^{\infty} \frac{|u(x)-u(y)|^{p}}{|x-y|^{1+\lambda p}} d x d y .
$$

Notice that the additional term $u(0)$ at the left hand side of (1.6) is essential since the integral on the left hand side of (1.5) diverges if $u$ is continuous at zero, $u(0) \neq 0$ and $\lambda>\frac{1}{p}$. 
The inequality (1.5) was extended in the seventies by Kufner and Triebel [5]: Roughly speaking, they derived - instead of (1.2) - the inequality

$$
\|u\|_{p, w_{0}} \leq C\left(\left\|u^{(\lambda)}\right\|_{p, w_{\lambda}}+\|u\|_{p, \tilde{w}}\right)
$$

where now

$$
\left\|u^{(\lambda)}\right\|_{p, w_{\lambda}}=\left(\int_{0}^{\infty} \int_{0}^{\infty} \frac{|u(x)-u(y)|^{p}}{|x-y|^{1+\lambda p}} w_{\lambda}(x) d x d y\right)^{1 / p},
$$

$\widetilde{w}$ is a certain additional weight and

$$
w_{0}(x)=w_{\lambda}(x) \cdot x^{-\lambda p} .
$$

The double integral in (1.7) offers one possibility of characterizing the still undetermined expression for $\left\|u^{(\lambda)}\right\|_{p, w_{\lambda}}$. But in spite of the symmetry of the "non-weighted" expression for $\left\|u^{(\lambda)}\right\|_{s}$ in (1.4), we would prefer a certain symmetry in $x$ and $y$ also in the weighted case, i.e., we are looking for a general inequality of the form

$$
\int_{0}^{\infty}|u(x)|^{p} w_{0}(x) d x \leq C^{p} \int_{0}^{\infty} \int_{0}^{\infty} \frac{|u(x)-u(y)|^{p}}{|x-y|^{1+\lambda p}} W(x, y) d x d y,
$$

possibly (but not necessarily) with $W(x, y)=W(y, x)$. In Section 4 of this paper we will prove some new inequalities of the type (1.9).

Our investigations were motivated by results of Kufner and Persson [4] and Burenkov and Evans [1]. In [4] e.g. the case when $W(x, y)=w(x)$ or $W(x, y)=w(y)$ is handled, and in [1], the case when $W(x, y)=$ $w(|x-y|)|x-y|^{1+\lambda p}$ is treated. For the reader's convenience and for later purposes, some of these results are briefly discussed and compared in Section 2.

Up to now, we dealt with the case

$$
p=q
$$

and all inequalities mentioned above can be derived more or less directly. Another approach using the theory of interpolation of Banach spaces was used in [4] and led to an inequality of the type 


$$
\begin{aligned}
& \left(\int_{0}^{\infty}|u(x)|^{q} w_{0}(x) d x\right)^{1 / q} \\
& \quad \leq C\left(\int_{0}^{\infty}\left(\int_{0}^{\infty} \frac{|u(x)-u(y)|^{p}}{|x-y|^{1+\lambda p}} w(x) d x\right)^{q / p} d y\right)^{1 / q}
\end{aligned}
$$

i.e., to an expression with a mixed norm on the right hand side for $p \neq q$. Moreover, it was supposed that $1<p \leq q<\infty$ [while the case $q<p$ is still open] and the authors have not been able to remove the mixed norm for $p \neq q$. In Section 3, we will prove a new inequality of the type (1.10), moreover, with a measure $v(y) d y$ instead of $d y$, but again only for $p \leq q$.

Some of the results obtained in this paper can be extended in various directions. Here we only present and prove an Orlicz norm version of the inequality (1.5) [and of its extension to the power weight case - see, e.g., (2.6) with $\beta=1+\lambda p$ ] and give an example of a multidimensional fractional order Hardy inequality; see Sections 5 and 6, respectively.

We close this introductory section by noting that in all cases we have considered here we have found that the "fractional" weights $w_{0}$ in the left integral are always of the type (1.8) for some suitable weight $w_{\lambda}$. Maybe this is supported by the following inequality which is an easy consequence of (1.5) and of the fact that $u$ belongs to the weighted space $L^{p}(w)$ if and only if $u w^{1 / p}$ belongs to the (non-weighted) space $L^{p}$ :

$$
\begin{aligned}
& \int_{0}^{\infty}|u(x)|^{p} w(x) x^{-\lambda p} d x \\
& \quad \leq C \int_{0}^{\infty} \int_{0}^{\infty} \frac{\left|u(x) w^{1 / p}(x)-u(y) w^{1 / p}(y)\right|^{p}}{|x-y|^{1+\lambda p}} d x d y .
\end{aligned}
$$

Also the right hand side in (1.11) could serve as a definition of the expression $\left\|u^{(\lambda)}\right\|_{p, w}$.

\section{SOME PRELIMINARY RESULTS AND DISCUSSIONS}

For the special case when the weight function $W(x, y)$ in the right hand side of (1.9) depends on $|x-y|$, Burenkov and Evans [1] recently proved the following interesting result: 
TheOREM 2.1 Let $0<p<\infty$, let $w$ be a weight function on $(0, \infty)$ and define

$$
v(x):=\int_{x}^{\infty} w(t) d t
$$

Suppose that $v$ satisfies the $\Delta_{2}$-condition, i.e., there exists a constant $c>0$ such that

$$
v(2 t) \leq c v(t) \text { for all } t>0 .
$$

Then for all $u \in L^{p}(0, \infty ; v)$

$$
\int_{0}^{\infty}|u(x)|^{p} v(x) d x \leq C^{p} \int_{0}^{\infty} \int_{0}^{\infty}|u(x)-u(y)|^{p} w(|x-y|) d x d y .
$$

For later purposes we also state the following slight improvement of the recent result by Kufner and Persson mentioned in the introduction:

THEOREM 2.2 Let $1<p<\infty$ and $\lambda \geq-1 / p$. Furthermore, assume that the function $u$ satisfies

$$
\lim _{x \rightarrow \infty} \frac{1}{x} \int_{0}^{x} u(t) d t=0 .
$$

Let $w_{0}$ and $w_{1}$ be weight functions on $(0, \infty)$ satisfying

$$
B:=\sup _{x>0}\left(\int_{0}^{x} w_{0}(t) d t\right)\left(\int_{x}^{\infty} w_{1}^{1-p^{\prime}}(t) d t\right)^{p-1}<\infty
$$

with $p^{\prime}=\frac{p}{p-1}$. Then, for every $\beta \geq 0$,

$$
\int_{0}^{\infty}|u(x)|^{p} w_{0}(x) d x \leq C^{p} \int_{0}^{\infty} \int_{0}^{x} \frac{|u(x)-u(y)|^{p}}{|x-y|^{\beta}} W(x) d y d x
$$

where

$$
W(x)=x^{\beta-1} w_{0}(x)+x^{\beta-1-p} w_{1}(x)
$$

and $C^{p}=2^{p-1} \max \left(1, C_{p}\right)$ with $C_{p} \leq B \cdot p^{p}(p-1)^{1-p}$.

The proof of Theorem 2.1 is similar to that of Proposition 1 in [4] but for the reader's convenience we present here the details. 
Proof First we use an idea by Grisvard [2] (cf. also p. 261 in Triebel [9]) and define

$$
g(x)=u(x)-\frac{1}{x} \int_{0}^{x} u(y) d y=\frac{1}{x} \int_{0}^{x}[u(x)-u(y)] d y .
$$

Obviously, $g(\infty)=u(\infty)$ and

$$
u^{\prime}(y)=u^{\prime}(y)-\frac{u(y)}{y}+\frac{1}{y^{2}} \int_{0}^{y} u(x) d x+\frac{g(y)}{y}=\left(g(y)-\int_{y}^{\infty} \frac{g(x)}{x} d x\right)^{\prime}
$$

and we conclude that

$$
u(y)=g(y)-\int_{y}^{\infty} \frac{g(x)}{x} d x .
$$

Therefore by using the inequality $|a+b|^{p} \leq 2^{p-1}\left(|a|^{p}+|b|^{p}\right)$, the assumption (2.1) and Hardy's inequality (see, e.g., [7]) we obtain that

$$
\begin{aligned}
\int_{0}^{\infty} & |u(x)|^{p} w_{0}(x) d x \\
& \leq 2^{p-1}\left(\int_{0}^{\infty}|g(x)|^{p} w_{0}(x) d x+\int_{0}^{\infty} w_{0}(x)\left|\int_{x}^{\infty} \frac{g(y)}{y} d y\right|^{p} d x\right) \\
& \leq 2^{p-1}\left(\int_{0}^{\infty}|g(x)|^{p} w_{0}(x) d x+C_{p} \int_{0}^{\infty} w_{1}(x)\left|\frac{g(x)}{x}\right|^{p} d x\right) \\
& =2^{p-1} \int_{0}^{\infty}|g(x)|^{p} W_{0}(x) d x
\end{aligned}
$$

where $W_{0}(x)=w_{0}(x)+C_{p} x^{-p} w_{1}(x)$. Therefore, by denoting $W_{1}(x)=$ $w_{0}(x)+x^{-p} w_{1}(x)$ and using (2.3) we find that

$$
\begin{gathered}
\int_{0}^{\infty}|u(x)|^{p} w_{0}(x) d x \leq C \int_{0}^{\infty}|g(x)|^{p} W_{1}(x) d x \leq \\
\quad \leq C \int_{0}^{\infty}\left(\frac{1}{x} \int_{0}^{x}|u(x)-u(y)| d y\right)^{p} W_{1}(x) d x .
\end{gathered}
$$

Furthermore, by Hölder's inequality and the assumption $\beta \geq 0$, we find that 


$$
\begin{aligned}
\left(\frac{1}{x} \int_{0}^{x}|u(x)-u(y)| d y\right)^{p} & \leq x^{-p} x^{p-1} \int_{0}^{x}|u(x)-u(y)|^{p} d y \\
& =x^{\beta-1} \int_{0}^{x} \frac{|u(x)-u(y)|^{p}}{x^{\beta}} d y \\
& \leq x^{\beta-1} \int_{0}^{x} \frac{|u(x)-u(y)|^{p}}{|x-y|^{\beta}} d y .
\end{aligned}
$$

The estimate (2.2) follows by combining (2.4) and (2.5).

Corollary 2.3 Let $1<p<\infty, \beta \geq 0, \lambda \geq-1 / p$ and $\alpha>\lambda p-1$. If $\lim _{x \rightarrow \infty} \frac{1}{x} \int_{0}^{x} u(t) d t=0$, then

$$
\int_{0}^{\infty}|u(x)|^{p} x^{\alpha-\lambda p} d x \leq C^{p} \int_{0}^{\infty} \int_{0}^{x} \frac{|u(x)-u(y)|^{p}}{|x-y|^{\beta}} x^{\beta-1-\lambda p+\alpha} d y d x .
$$

Proof Apply Theorem 2.2 with $w_{0}(x)=x^{\alpha-\lambda p}$ and $w_{1}(x)=x^{\alpha-\lambda p+p}$.

Let us note that applying Theorem 2.1 with $w(t)=t^{\alpha-\lambda p-1}$ we find that for $\alpha<\lambda p$ we have

$$
\int_{0}^{\infty}|u(x)|^{p} x^{\alpha-\lambda p} d x \leq C^{p} \int_{0}^{\infty} \int_{0}^{\infty} \frac{|u(x)-u(y)|^{p}}{|x-y|^{1+\lambda p}}|x-y|^{\alpha} d x d y .
$$

Moreover, Theorem 2.1 cannot be used for any $\alpha \geq \lambda p$ (since then $v(x) \equiv \infty)$. But using Corollary 2.3 we see that (2.7) holds also if $\lambda p \leq \alpha<\lambda p+1$ : This fact follows from (2.6) putting there $\beta=0$ and noting that $x^{-1-\lambda p+\alpha} \leq|x-y|^{-1-\lambda p+\alpha}$ for all $y, 0<y \leq x$ and $-1-\lambda p+\alpha<0$.

More generally, using Theorem 2.2 with $\beta=0$ and with $W(x)$ strictly decreasing we obtain an inequality of the type

$$
\int_{0}^{\infty}|u(x)|^{p} w_{0}(x) d x \leq C^{p} \int_{0}^{\infty} \int_{0}^{\infty}|u(x)-u(y)|^{p} W(|x-y|) d x d y,
$$

and this inequality cannot be obtained in general using Theorem 2.1 e.g. in the case that the integral $\int_{x}^{\infty} w(t) d t$ is divergent. Another inequality of the type (2.8) can be obtained by using our Theorem 4.1 with $\omega(x, y)=w(|x-y|)$ (see Remark 4.5). 


\section{THE GENERAL (MIXED NORM) CASE $p \neq q$}

Let $w$ and $v$ denote weight functions on $(0, \infty)$. Moreover, define

$$
\begin{aligned}
V(y): & =\int_{0}^{y} v(x) d x \\
\omega_{p, q}(y): & =\left(\frac{1}{y} \int_{0}^{y}\left(\frac{v^{p}(x)}{w(x)}\right)^{\frac{1}{p-1}} d x\right)^{-\frac{q}{p^{\prime}}}\left(\frac{1}{y} \int_{0}^{y} v(x) d x\right)^{q} v(y), \\
(\Delta u)_{\lambda}(x, y): & =\frac{|u(x)-u(y)|}{|x-y|^{\lambda}} .
\end{aligned}
$$

The main result of this section reads as follows:

TheOREM 3.1 Let $1<p \leq q<\infty, \lambda \geq-1 / p, w_{\lambda}(x)=\omega_{p, q}(x) x^{-\lambda q}$ and

$$
\begin{aligned}
C_{p, q}:=\sup _{r>0} & \left(\int_{r}^{\infty} w_{\lambda}(x) V^{-q}(x) d x\right)^{1 / q} . \\
& \cdot\left(\int_{0}^{r}\left[w_{\lambda}(x) v^{-q}(x)\right]^{1-q^{\prime}} d x\right)^{1 / q^{\prime}}<\infty .
\end{aligned}
$$

Then for $u \in L^{q}\left(0, \infty ; w_{\lambda}\right)$

$$
\begin{aligned}
& \left(\int_{0}^{\infty}|u(x)|^{q} w_{\lambda}(x) d x\right)^{1 / q} \\
& \quad \leq \frac{1}{1-K}\left(\int_{0}^{\infty}\left[\int_{0}^{\infty} \frac{\left|\left(\Delta u_{\lambda}\right)(x, y)\right|^{p}}{|x-y|} w(x) d x\right]^{q / p} v(y) d y\right)^{1 / q},
\end{aligned}
$$

provided

$$
K=\frac{C_{p, q} q}{(q-1)^{1 / q^{\prime}}}<1
$$

Remark 3.2 Note that for the case $v(y) \equiv 1$ we obtain the mixed norm inequality (1.10) with

$$
w_{0}(x)=w_{\lambda}(x)=x^{\left(-\lambda+1 / p^{\prime}\right) q}\left(\int_{0}^{x} w^{1-p^{\prime}}(t) d t\right)^{-q / p^{\prime}} .
$$


Proof Hölder's inequality yields

$$
\begin{aligned}
& \left(\int_{0}^{y}|u(x)|-u(y) \mid v(x) d x\right)^{q} \\
& \quad \leq\left(\int_{0}^{y}|u(x)-u(y)|^{p} w(x) d x\right)^{q / p}\left(\int_{0}^{y}\left(\frac{v^{p}(x)}{w(x)}\right)^{\frac{1}{p-1}} d x\right)^{q / p^{\prime}} .
\end{aligned}
$$

Therefore

$$
\begin{aligned}
\int_{0}^{\infty} & \left(\int_{0}^{\infty} \frac{\left|(\Delta u)_{\lambda}(x, y)\right|^{p}}{|x-y|} w(x) d x\right)^{q / p} v(y) d y \\
\geq & \int_{0}^{\infty}\left(\int_{0}^{y} \frac{|u(x)-u(y)|^{p}}{y^{1+\lambda p}} w(x) d x\right)^{q / p} v(y) d y \\
\geq & \int_{0}^{\infty} y^{-q / p-\lambda q}\left(\int_{0}^{y}\left(\frac{v^{p}(x)}{w(x)}\right)^{\frac{1}{p-1}} d x\right)^{-q / p^{\prime}} . \\
& \cdot\left(\int_{0}^{y}|u(x)-u(y)| v(x) d x\right)^{q} v(y) d y \\
\geq & \int_{0}^{\infty} y^{-q / p-\lambda q}\left(\int_{0}^{y}\left(\frac{v^{p}(x)}{w(x)}\right)^{\frac{1}{p-1}} d x\right)^{-q / p^{\prime}} \mid u(y) \int_{0}^{y} v(x) d x \\
& -\left.\int_{0}^{y} u(x) v(x) d x\right|^{q} v(y) d y \\
= & \int_{0}^{\infty} w_{\lambda}(y)\left|u(y)-\frac{1}{V(y)} \int_{0}^{y} u(x) v(x) d x\right|^{q} d y .
\end{aligned}
$$

Using this estimate together with the Minkowski and Hardy inequalities [the latter one can be used due to (3.1)] we find that

$$
\begin{aligned}
& \left(\int_{0}^{\infty}|u(y)|^{q} w_{\lambda}(y) d y\right)^{1 / q} \\
& \quad \leq\left(\int_{0}^{\infty} w_{\lambda}(y)\left|u(y)-\frac{1}{V(y)} \int_{0}^{y} u(x) v(x) d x\right|^{q} v(y) d y\right)^{1 / q} \\
& \quad+\left(\int_{0}^{\infty} w_{\lambda}(y) V^{-q}(y)\left(\int_{0}^{y}|u(x)| v(x) d x\right)^{q} d y\right)^{1 / q}
\end{aligned}
$$




$$
\begin{aligned}
& \leq\left(\int_{0}^{\infty}\left(\int_{0}^{\infty} \frac{\left|\left(\Delta u_{\lambda}\right)(x, y)\right|^{p}}{|x-y|} w(x) d x\right)^{q / p} v(y) d y\right)^{1 / q} \\
& +K\left(\int_{0}^{\infty} w_{\lambda}(y)|u(y)|^{q} d y\right)^{1 / q} .
\end{aligned}
$$

Inequality (3.2) follows by subtracting and using (3.3).

Considering the case $p=q$ in Theorem 3.1 we obtain

Corollary 3.3 If $1<p<\infty, \lambda \geq-1 / p, w_{\lambda}(x)=\omega_{p, p}(x) x^{-\lambda p}$ and

$$
\begin{aligned}
C_{p}:=\sup _{r>0} & \left(\int_{r}^{\infty} w_{\lambda}(x) V^{-p}(x) d x\right)^{1 / p} . \\
& \cdot\left(\int_{0}^{r}\left(w_{\lambda}(x) v^{-p}(x)\right)^{1-p^{\prime}} d x\right)^{1 / p^{\prime}}<\infty,
\end{aligned}
$$

then

$$
\begin{aligned}
& \left(\int_{0}^{\infty}|u(x)|^{p} w_{\lambda}(x) d x\right)^{1 / p} \\
& \quad \leq \frac{1}{1-K}\left(\int_{0}^{\infty} \int_{0}^{\infty} \frac{|u(x)-u(y)|^{p}}{|x-y|^{1+\lambda p}} w(x) v(y) d x d y\right)^{1 / p}
\end{aligned}
$$

provided

$$
K=\frac{C_{p} p}{(p-1)^{1 / p^{\prime}}}<1 .
$$

Consequently, (3.4) may be regarded as a fractional order Hardy inequality of the type (1.9) with the weight

$$
W(x, y)=w(x) v(y)
$$

on the right hand side.

Remark 3.4 Applying Corollary 3.3 with $w(x) \equiv 1, v(y) \equiv 1$ and with $u(x)$ replaced by $u(x)-u(0)$ we find that if $1<p<\infty, \lambda>1 / p$, then

$$
\begin{aligned}
& \left(\int_{0}^{\infty}|u(x)-u(0)|^{p} x^{-\lambda p} d x\right)^{1 / p} \\
& \quad \leq \frac{\lambda p+p-1}{\lambda p-1}\left(\int_{0}^{\infty} \int_{0}^{\infty} \frac{|u(x)-u(y)|^{p}}{|x-y|^{1+\lambda p}} d x d y\right)^{1 / p},
\end{aligned}
$$


cf. formula (1.6). Moreover, it is not difficult to see that (3.5) holds with $0<\lambda<1$ and with the constant $\frac{1}{2} \lambda /(1-\lambda)$ for $p=1$.

Remark 3.5 Theorem 3.1 requires a certain integrability of $v$ and $\left(v^{p} w^{-1}\right)^{\frac{1}{p-1}}$ on $(0, y)$ for $y>0$. This requirement can be replaced by an integrability condition on $(y, \infty)$, if one modifies the proof of Theorem 3.1 as follows: Let

$$
\widehat{V}(y):=\int_{y}^{\infty} v(x) x^{-1 / p-\lambda-\delta} d x
$$

with some real parameter $\delta$, and

$$
\widehat{w}_{\lambda}(y):=v(y)\left(\int_{y}^{\infty}\left(\frac{v^{p}(x)}{w(x)}\right)^{\frac{1}{p-1}} x^{-\delta p^{\prime}} d x\right)^{-q / p^{\prime}} \widehat{V}^{q}(y) .
$$

Now, we use the estimate

$$
\begin{aligned}
\int_{0}^{\infty} & \left(\int_{0}^{\infty} \frac{\left|(\Delta u)_{\lambda}(x, y)\right|^{p}}{|x-y|} w(x)\right)^{q / p} v(y) d y \\
& \geq \int_{0}^{\infty}\left(\int_{y}^{\infty} \frac{|u(x)-u(y)|^{p}}{x^{1+\lambda p}} w(x) d x\right)^{q / p} v(y) d y
\end{aligned}
$$

and denote the last double integral by $J$. Since Hölder's inequality yields

$$
\begin{aligned}
& \left(\int_{y}^{\infty} \frac{|u(x)-u(y)|}{x^{1 / p+\lambda+\delta}} v(x) d x\right)^{q} \\
& \leq\left(\int_{y}^{\infty} \frac{|u(x)-u(y)|^{p}}{x^{1+\lambda p}} w(x) d x\right)^{q / p}\left(\int_{y}^{\infty}\left(\frac{v^{p}(x)}{w(x)}\right)^{\frac{1}{p-1}} x^{-\delta p^{\prime}} d x\right)^{q / p^{\prime}}
\end{aligned}
$$

we obtain that

$$
\begin{aligned}
J \geq & \int_{0}^{\infty} v(y)\left(\int_{y}^{\infty}\left(\frac{v^{p}(x)}{w(x)}\right)^{\frac{1}{p-1}} x^{-\delta p^{\prime}} d x\right)^{-q / p} . \\
& \cdot\left|u(y) \int_{y}^{\infty} v(x) x^{-1 / p-\lambda-\delta} d x-\int_{y}^{\infty} u(x) v(x) x^{-1 / p-\lambda-\delta} d x\right|^{q} d y \\
= & \int_{0}^{\infty} \widehat{w}_{\lambda}(y)\left|u(y)-\frac{1}{\widehat{V}(y)} \int_{y}^{\infty} u(x) v(x) x^{-1 / p-\lambda-\delta} d x\right|^{q} d y .
\end{aligned}
$$


Then we proceed as in the proof of Theorem 3.1, using the Minkowski inequality and the Hardy inequality - the last one assuming that $\widehat{C}_{p, q}<\infty$ where

$$
\begin{aligned}
\widehat{C}_{p, q}:=\sup _{r>0} & \left(\int_{0}^{r} \widehat{w}_{\lambda}(x) \widehat{V}^{-q}(x) d x\right)^{1 / q} . \\
& \cdot\left(\int_{r}^{\infty}\left(\widehat{w}_{\lambda}(x) v^{-q}(x) x^{q / p+\lambda q+\delta q}\right)^{1-q^{\prime}} d x\right)^{1 / q^{\prime}} .
\end{aligned}
$$

Finally, we obtain again inequality (3.2) - with $w_{\lambda}(x)$ replaced by $\widehat{w}_{\lambda}(x)-$ provided

$$
K=\frac{\widehat{C}_{p, q} q}{(q-1)^{1 / p^{\prime}}}<1 .
$$

\section{A GENERAL WEIGHT IN TWO VARIABLES FOR $p=q$}

In this section, we will prove the following assertion:

THEOREM 4.1 Let $\omega(x, y)$ be a non-negative measurable function on $(0, \infty) \times$ $(0, \infty)$, locally integrable in both variables separately. Let $1<p<\infty$ and $\lambda \geq-1 / p$.

(i) Denote

$$
W(x)=\left(\frac{1}{x} \int_{0}^{x} \omega^{1-p^{\prime}}(x, t) d t\right)^{1-p}
$$

and $W_{\lambda}(x)=W(x) x^{-\lambda p}$. If

$$
C_{p}:=\sup _{r>0}\left(\int_{r}^{\infty} \frac{W(x)}{x^{p(\lambda+1)}} d x\right)^{1 / p}\left(\int_{0}^{r} W^{1-p^{\prime}}(x) x^{\lambda p^{\prime}} d x\right)^{1 / p^{\prime}}<\infty
$$

and

$$
K=\frac{C_{p} p}{(p-1)^{1 / p^{\prime}}}<1,
$$

then for $u \in L^{p}\left(0, \infty ; W_{\lambda}\right)$

$$
\begin{aligned}
\left(\int_{0}^{\infty} W_{\lambda}(x)\right. & \left.|u(x)|^{p} d x\right)^{1 / p} \\
\leq & \frac{1}{1-K}\left(\int_{0}^{\infty} \int_{0}^{\infty} \frac{|u(x)-u(y)|^{p}}{|x-y|^{1+\lambda p}} \omega(x, y) d x d y\right)^{1 / p} .
\end{aligned}
$$


(ii) Denote

$$
\bar{W}(y)=\left(\frac{1}{y} \int_{0}^{y} \omega^{1-p^{\prime}}(t, y) d t\right)^{1-p}
$$

and $\bar{W}_{\lambda}(x)=\bar{W}(x) x^{-\lambda p}$. If

$$
\bar{C}_{p}:=\sup _{r>0}\left(\int_{r}^{\infty} \frac{\bar{W}(y)}{y^{p(\lambda+1)}} d y\right)^{1 / p}\left(\int_{0}^{r} \bar{W}^{1-p^{\prime}}(y) y^{\lambda p^{\prime}} d y\right)^{1 / p^{\prime}}<\infty
$$

and

$$
\bar{K}=\frac{\bar{C}_{p} p}{(p-1)^{1-p^{\prime}}}<1
$$

then (4.2) holds with $W_{\lambda}$ and $K$ replaced by $\bar{W}_{\lambda}$ and $\bar{K}$, respectively.

Proof Obviously

$$
\begin{aligned}
\int_{0}^{\infty} \int_{0}^{\infty} & \frac{|u(x)-u(y)|^{p}}{|x-y|^{1+\lambda p}} \omega(x, y) d x d y \\
= & \int_{0}^{\infty} \int_{0}^{x} \frac{|u(x)-u(y)|^{p}}{|x-y|^{1+\lambda p}} \omega(x, y) d y d x \\
& +\int_{0}^{\infty} \int_{x}^{\infty} \frac{|u(x)-u(y)|^{p}}{|x-y|^{1+\lambda p}} \omega(x, y) d y d x \\
\geq & \int_{0}^{\infty} \int_{0}^{x} \frac{|u(x)-u(y)|^{p}}{x^{1+\lambda p}} \omega(x, y) d y d x \\
& +\int_{0}^{\infty} \int_{x}^{\infty} \frac{|u(x)-u(y)|^{p}}{y^{1+\lambda p}} \omega(x, y) d y d x=I_{1}+I_{2}
\end{aligned}
$$

Hölder's inequality yields

$$
\begin{aligned}
\left|\int_{0}^{x}(u(x)-u(y)) d y\right|^{p} \leq & \left(\int_{0}^{x}|u(x)-u(y)|^{p} \omega(x, y) d y\right) . \\
& \cdot\left(\int_{0}^{x} \omega^{1-p^{\prime}}(x, t) d t\right)^{p-1},
\end{aligned}
$$


and consequently

$$
\begin{aligned}
I_{1} & \geq \int_{0}^{\infty} \frac{W(x) x^{1-p}}{x^{1+\lambda p}}\left|\int_{0}^{x}(u(x)-u(y)) d y\right|^{p} \\
& =\int_{0}^{\infty} \frac{W(x) x^{1-p}}{x^{1+\lambda p}}\left|x u(x)-\int_{0}^{x} u(y) d y\right|^{p} \\
& =\int_{0}^{\infty} \frac{W(x)}{x^{\lambda p}}\left|u(x)-\frac{1}{x} \int_{0}^{x} u(y) d y\right|^{p} .
\end{aligned}
$$

Hence, by the Minkowski and Hardy inequalities, we have

$$
\begin{aligned}
\left(\int_{0}^{\infty} W(x)\right. & \left.x^{-\lambda p}|u(x)|^{p} d x\right)^{1 / p} \\
\leq & \left(\int_{0}^{x} \frac{W(x)}{x^{\lambda p}}\left|u(x)-\frac{1}{x} \int_{0}^{x} u(y) d y\right|^{p} d x\right)^{1 / p} \\
& +\left(\left.\int_{0}^{x} \frac{W(x)}{x^{\lambda p+p} \mid} \int_{0}^{x} u(y) d y\right|^{p} d x\right)^{1 / p} \\
\leq & I_{1}^{1 / p}+K\left(\int_{0}^{\infty} W(x) x^{-\lambda p}|u(x)|^{p} d x\right)^{1 / p}
\end{aligned}
$$

and since $K<1$, (4.2) follows at once.

Part (ii) can be proved completely analogously, estimating now from below the integral $I_{2}$, which can be rewritten by Fubini's theorem as

$$
I_{2}=\int_{0}^{\infty} \int_{0}^{y} \frac{|u(x)-u(y)|^{p}}{y^{1+\lambda p}} \omega(x, y) d x d y
$$

But it follows also directly from part (i) using the following symmetry argument: Since for $h(x, y)=|u(x)-u(y)|^{p}|x-y|^{-1-\lambda p}$ we have $h(x, y)=h(y, x)$, the right hand side in (4.2) satisfies

$$
\int_{0}^{\infty} \int_{0}^{\infty} h(x, y) w(x, y) d x d y=\int_{0}^{\infty} \int_{0}^{\infty} h(x, y) w(y, x) d x d y
$$

If we denote for $w_{1}(x, y)=w(y, x)$ by $W_{1}(x)$ the function, which corresponds to $w_{1}$ as $W$ corresponds to $w$, we have that $W_{1}(x)=\bar{W}(x)$, and now we proceed as in the proof of part (i) with $W_{1}$ instead of $W$. 
Remark 4.2 The proof of Theorem 4.1 shows that in fact we can derive a better inequality provided (4.1) and (4.3) hold simultaneously:

$$
\begin{aligned}
& (1-K)\left(\int_{0}^{\infty} W_{\lambda}(x)|u(x)|^{p} d x\right)^{1 / p} \\
& \quad+(1-\bar{K})\left(\int_{0}^{\infty} \bar{W}_{\lambda}(x)|u(x)|^{p} d x\right)^{1 / p} \\
& \quad \leq\left(\int_{0}^{\infty} \int_{0}^{\infty} \frac{|u(x)-u(y)|^{p}}{|x-y|^{1+\lambda p}} \omega(x, y) d x d y\right)^{1 / p} .
\end{aligned}
$$

Corollary 4.3 Let $1<p<\infty, \lambda \geq-1 / p$ and $\alpha<\lambda p-1$. Then

$$
\begin{aligned}
& \left(\int_{0}^{\infty}|u(x)-u(0)|^{p} x^{\alpha-\lambda p} d x\right)^{1 / p} \\
& \quad \leq \frac{\lambda p+p-\alpha-1}{\lambda p-\alpha-1}\left(\int_{0}^{\infty} \int_{0}^{\infty} \frac{|u(x)-u(y)|^{p}}{|x-y|^{1+\lambda p}} x^{\alpha} d x d y\right)^{1 / p} .
\end{aligned}
$$

Proof Apply Theorem 4.1 (i) with $u(x)$ replaced by $u(x)-u(0)$ and with $\omega(x, y)=w(x)=x^{\alpha}$. Then $W(x)=x^{\alpha}$ and (4.1) holds with $C_{p}=(p-1)^{1 / p^{\prime}} /(\lambda p+p-\alpha-1)$ provided $\alpha-p \lambda-p<-1$ and $-\alpha p^{\prime} / p+\lambda p^{\prime}+1>0$, i.e., if $\alpha+1<p(\lambda+1)$. But this is the case if $\alpha+1<\lambda p$. Moreover, $K=p /(\lambda p+p-\alpha-1)<1$ if $\alpha+1<\lambda p$.

Remark 4.4 For the case $\alpha=0$, the statement in Corollary 4.3 follows also at once from our Corollary 3.3 (see Remark 3.4). Moreover, according to Corollary 1 in [4] (cf. our Theorem 2.2), the following complement also holds: If $1<p<\infty, \lambda \geq-1 / p$ and $\alpha>\lambda p-1$, then

$$
\left(\int_{0}^{\infty}|u(x)|^{p} x^{\alpha-\lambda p} d x\right)^{1 / p} \leq C\left(\int_{0}^{\infty} \int_{0}^{\infty} \frac{|u(x)-u(y)|^{p}}{|x-y|^{1+\lambda p}} x^{\alpha} d x d y\right)^{1 / p}
$$

where $C=2^{p-1}(1+p /(\alpha-\lambda p+1))$. For $\alpha=0$, the inequalities (4.4) and (4.5) coincide with the inequalities (1.6) and (1.5) mentioned in Section 1.

Remark 4.5 By applying Theorem 4.1 with $\omega(x, y)=w(|x-y|)|x-y|^{-1-\lambda p}$ we obtain another inequality of the Burenkov-Evans type (see Theorem 2.1). In particular by using this result with $w(x)=x^{\alpha}$ we rediscover the inequality (2.7). 
Remark 4.6 In Theorem 4.1, we in fact used the integrability of $\omega^{1-p^{\prime}}(x, y)$ either with respect to $x$ or with respect to $y$ on intervals $(0, z), z>0$. If this condition is not fulfilled, we can proceed in a similar way as in Remark 3.5: We estimate the integral $I_{2}$ from the proof of Theorem 4.1,

$$
I_{2}=\int_{0}^{\infty} \int_{x}^{\infty} \frac{|u(x)-u(y)|^{p}}{y^{1+\lambda p}} \omega(x, y) d y d x
$$

from below using the following Hölder inequality:

$$
\begin{aligned}
& \mid \int_{x}^{\infty} \frac{u(x)-u(y)}{\left.y^{1 / p+\lambda+\delta} \omega(x, y) d y\right|^{p}} \\
& \quad \leq\left(\int_{x}^{\infty} \frac{|u(x)-u(y)|^{p}}{y^{1+\lambda p}} \omega(x, y) d y\right)\left(\int_{x}^{\infty} y^{-\delta p^{\prime}} \omega(x, y) d y\right)^{p-1}
\end{aligned}
$$

with a suitable parameter $\delta$. Here, the role of the function $W(x)$ is played by

$$
W^{*}(x)=\left(\int_{x}^{\infty} y^{-\delta p^{\prime}} \omega(x, y) d y\right)^{1-p}
$$

i.e., we need the integrability of $\omega(x, y) y^{-\delta p^{\prime}}$ with some $\delta$ in the neighbourhood of infinity. The remaining steps are similar as in the proof of Theorem 4.1 (i) and are left to the reader (see also Remark 3.5). Let us mention that condition (4.1) is then replaced by

$$
\begin{aligned}
C_{p}^{*}:=\sup _{r>0} & \left(\int_{0}^{r} W^{*}(x) d x\right)^{1 / p} . \\
& \cdot\left(\int_{0}^{\infty} W^{* 1-p^{\prime}}(x) \cdot x^{1 / p^{\prime}-p^{\prime}+\lambda+\delta} d x\right)^{1 / p^{\prime}}<\infty,
\end{aligned}
$$

the function $W_{\lambda}(x)=W(x) x^{-\lambda p}$ in (4.2) is replaced by $W^{*}(x) \cdot x^{1-1 / p-\lambda-\delta}$ and the parameter $\delta$ has to satisfy $\delta>1-1 / p-\lambda$.

A similar result can be obtained using the integrability of $x^{-\delta p^{\prime}} \omega(x, y)$ (as a function of $x$ !) in the neighbourhood of infinity if we proceed analogously with $I_{1}$ rewritten (by Fubini's theorem) as

$$
I_{1}=\int_{0}^{\infty} \int_{y}^{\infty} \frac{|u(x)-u(y)|^{p}}{x^{1+\lambda p}} \omega(x, y) d x d y .
$$




\section{A GENERALIZATION TO THE ORLICZ NORM}

In this section we will modify inequalities (1.5) and (2.6), i.e., the case with power weights $x^{\alpha}$, using the norm in a suitable Orlicz space.

For this purpose, let $P$ be a Young function satisfying the $\Delta_{2}$-condition. Then it is well-known that there exists a $\beta>0$ such that for all $\kappa>1$,

$$
P(\kappa t) \leq \kappa^{\beta} P(t)
$$

If $H$ is the Hardy (averaging) operator defined by

$$
(H u)(x):=\frac{1}{x} \int_{0}^{x} u(t) d t
$$

and both $P$ and its complementary function $\widehat{P}$ satisfy the $\Delta_{2}$-condition, then Palmieri [8] (cf. also [6, Corollary 4]) proved that

$$
\left\|t^{\nu} H u\right\|_{P} \leq \frac{\beta}{1-v \beta}\left\|t^{\nu} u\right\|_{P}
$$

where the norm in (5.2) is the Orlicz norm. [If $v<0$ then the condition that $\widehat{P} \in \Delta_{2}$ can be omitted.]

It is well known that the Orlicz norm $\|\cdot\|_{P}$ and the Luxemburg norm $\|\cdot\|_{P}^{*}$ defined by

$$
\|g\|_{P}^{*}:=\inf \left\{k>0: \int_{0}^{\infty} P\left(\frac{|g(x)|}{k}\right) d x \leq 1\right\}
$$

satisfy

$$
\|g\|_{P}^{*} \leq\|g\|_{P} \leq 2\|g\|_{P}^{*}
$$

Let $0<\lambda<1$ and denote

$$
v_{\lambda}(x)=\frac{v(x)}{x^{\lambda}},(\Delta u)_{\lambda}(x, y)=\frac{u(x)-u(y)}{|x-y|^{\lambda}}
$$

further, let $\|\cdot\|_{P(\mu)}$ denote the twodimensional Orlicz norm on $(0, \infty) \times$ $(0, \infty)$ with respect to the measure $d \mu=\frac{d x d y}{|x-y|}$.

The main result of this section reads as follows. 
THEOREM 5.1 If $\boldsymbol{P}$ and $\widehat{\boldsymbol{P}}$ satisfy (5.1) with the constant $\beta>0$, then for $\frac{\beta}{1+\lambda \beta}<1$,

$$
\left\|u_{\lambda}\right\|_{P} \leq C\left\|(\Delta u)_{\lambda}\right\|_{P(\mu)}
$$

where

$$
C=\frac{2(1+\beta \lambda)}{1+\beta(\lambda-1)}
$$

Moreover, if $\lambda>0$ the condition that $\widehat{P}$ satisfies (5.1) may be omitted.

Proof The convexity of $P$ yields

$$
\begin{aligned}
& \int_{0}^{\infty} \int_{0}^{\infty} P\left(\left|(\Delta u)_{\lambda}\right|\right) d \mu=\int_{0}^{\infty} \int_{0}^{\infty} P\left(\frac{|u(x)-u(y)|}{|x-y|^{\lambda}}\right) \frac{d x d y}{|x-y|} \\
& \geq \int_{0}^{\infty} \int_{0}^{x} P\left(\frac{|u(x)-u(y)|}{x^{\lambda}}\right) \frac{d y}{x} d x \\
& =\int_{0}^{\infty} \frac{1}{x} \int_{0}^{x} P\left(\frac{|u(x)-u(y)|}{x^{\lambda}}\right) d y d x \\
& \geq \int_{0}^{\infty} P\left(\frac{1}{x} \int_{0}^{x} \frac{|u(x)-u(y)|}{x^{\lambda}} d y\right) d x=\int_{0}^{\infty} P\left(\left|u_{\lambda}(x)-(H u)_{\lambda}(x)\right|\right) d x .
\end{aligned}
$$

The Minkowski inequality, the estimates (5.3) and the (Hardy) inequality (5.2) with $v=-\lambda$ yield

$$
\begin{aligned}
\left\|u_{\lambda}\right\|_{P} & =\left\|u_{\lambda}-(H u)_{\lambda}+(H u)_{\lambda}\right\|_{P} \\
& \leq\left\|u_{\lambda}-(H u)_{\lambda}\right\|_{P}+\left\|(H u)_{\lambda}\right\|_{P} \\
& \leq 2\left\|u_{\lambda}-(H u)_{\lambda}\right\|_{P}^{*}+\left\|t^{-\lambda} H u\right\|_{P} \\
& \leq 2\left\|(\Delta u)_{\lambda}\right\|_{P(\mu)}^{*}+\frac{\beta}{1+\beta \lambda}\left\|u_{\lambda}\right\|_{P} \\
& \leq 2\left\|(\Delta u)_{\lambda}\right\| P(\mu)+\frac{\beta}{1+\beta \lambda}\left\|u_{\lambda}\right\|_{P}
\end{aligned}
$$

and (5.4) follows immediately.

Of course (5.3) shows that (5.4) holds also with Orlicz norms replaced by the Luxemburg norms. 


\section{AN N-DIMENSIONAL FRACTIONAL ORDER HARDY INEQUALITY}

We are also able to prove some $N$-dimensional versions of the inequalities mentioned. First some notation: For $x \in \mathbb{R}^{N}, B(|x|)$ will denote the ball $\left\{y \in \mathbb{R}^{N} ;|y| \leq|x|\right\}$ and $|B(|x|)|$ its volume. It is $|B(|x|)|=|x|^{N}\left|S^{N-1}\right| / N$ where $S^{N-1}$ is the unit sphere in $\mathbb{R}^{N}$ and $\left|S^{N-1}\right|$ its area.

THeOREM 6.1 Let $1<p<\infty, N \geq 1$ and $\lambda p>1$. Then

$$
\begin{aligned}
& \left(\int_{\mathbb{R}^{N}} \frac{|u(x)|^{p}}{|x|^{\lambda p N}} d x\right)^{1 / p} \\
& \quad \leq \frac{2^{N(1+\lambda p) / p} N^{1 / p}}{\left|S^{N-1}\right|^{1 / p}} \frac{[p(1+\lambda)-1]}{\lambda p-1}\left(\int_{\mathbb{R}^{N}} \int_{\mathbb{R}^{N}} \frac{|u(x)-u(y)|^{p}}{|x-y|^{N(1+\lambda p)}} d y d x\right)_{(6.1)}^{1 / p} .
\end{aligned}
$$

Proof Obviously

$$
\begin{aligned}
J: & =\int_{\mathbb{R}^{N}} \int_{\mathbb{R}^{N}} \frac{|u(x)-u(y)|^{p}}{|x-y|^{N(1+\lambda p)}} d y d x \\
& \geq \int_{\mathbb{R}^{N}} \int_{B(|x|)} \frac{|u(x)-u(y)|^{p}}{|x-y|^{N(1+\lambda p)}} d y d x \\
& \geq \frac{1}{2^{N(1+\lambda p)}} \int_{\mathbb{R}^{N}}|x|^{-N(1+\lambda p)} \int_{B(|x|)}|u(x)-u(y)|^{p} d y d x
\end{aligned}
$$

since for $y \in B(|x|)$, it is $|x-y| \leq 2|x|$, and $1+\lambda p>0$. But Hölder's inequality yields

$$
\left|\int_{B(|x|)}(u(x)-u(y)) d y\right|^{p} \leq\left(\int_{B(|x|)}|u(x)-u(y)|^{p} d y\right)|B(|x|)|^{p-1},
$$

and consequently

$$
\begin{aligned}
J & \geq \frac{1}{2^{N(1+\lambda p)}} \int_{\mathbb{R}^{N}} \frac{|B(|x|)|^{1-p}}{|x|^{N(1+\lambda p)}}\left|\int_{B(|x|)}(u(x)-u(y)) d y\right|^{p} d x \\
& =\frac{1}{2^{N(1+\lambda p)}} \int_{\mathbb{R}^{N}} \frac{|B(|x|)|^{1-p}}{|x|^{N(1+\lambda p)}}|u(x)| B(|x|)\left|-\int_{B(|x|)} u(y) d y\right|^{p} d x \\
& =\frac{1}{2^{N(1+\lambda p)}} \int_{\mathbb{R}^{N}} \frac{B(|x|)}{|x|^{N(1+\lambda p)}}\left|u(x)-\frac{1}{|B(|x|)|} \int_{B(|x|)} u(y) d y\right|^{p} d x .
\end{aligned}
$$


Therefore, by Minkowski's inequality

$$
\begin{aligned}
& \left(\int_{\mathbb{R}^{N}} \frac{|u(x)|^{p}}{|x|^{\lambda p N}} d x\right)^{1 / p} \\
& =\left\{\int_{\mathbb{R}^{N}} \frac{1}{|x|^{\lambda p N}} \mid u(x)-\frac{1}{|B(|x|)|} \int_{B(|x|)} u(y) d y\right. \\
& \left.+\left.\frac{1}{|B(|x|)|} \int_{B(|x|)} u(y) d y\right|^{p} d x\right\}^{1 / p} \\
& \leq\left\{\int_{\mathbb{R}^{N}} \frac{1}{|x|^{\lambda p N}}\left|u(x)-\frac{1}{|B(|x|)|} \int_{B(|x|)} u(y) d y\right|^{p} d x\right\}^{1 / p} \\
& +\left\{\int_{\mathbb{R}^{N}} \frac{|B(|x|)|^{-p}}{|x|^{\lambda p N}}\left|\int_{B(|x|)} u(y) d y\right|^{p} d x\right\}^{1 / p} \equiv I_{1}+I_{2} .
\end{aligned}
$$

It follows from (6.2) that

$$
I_{1} \leq \frac{2^{N(1+\lambda p) / p} N^{1 / p}}{\left|S^{N-1}\right|^{1 / p}} J^{1 / p},
$$

and the Hardy inequality with power weights yields

$$
I_{2} \leq C_{p} \frac{p}{(p-1)^{1 / p^{\prime}}}\left(\int_{\mathbb{R}^{N}} \frac{|u(x)|^{p}}{|x|^{\lambda p N}} d x\right)^{1 / p}
$$

provided

$$
\begin{aligned}
C_{p}:= & \sup _{r>0}\left(\int_{|x| \geq r} \frac{|B(|x|)|^{-p}}{|x|^{\lambda p N}} d x\right)^{1 / p}\left(\int_{|x| \leq r}|x|^{-\lambda p N\left(1-p^{\prime}\right)} d x\right)^{1 / p^{\prime}} \\
= & \sup _{r>0}\left(\int_{S^{N-1}} \int_{r}^{\infty} t^{N-1-\lambda p N-N p} \frac{N^{p}}{\left|S^{N-1}\right| p} d t d \sigma\right)^{1 / p} \\
& \cdot\left(\int_{S^{N-1}} \int_{0}^{r} t^{N-1+\lambda p^{\prime} N} d t d \sigma\right)^{1 / p^{\prime}} \\
= & N\left|S^{N-1}\right|^{-1}\left|S^{N-1}\right|^{1 / p+1 / p^{\prime}} \sup _{r>0} \frac{r^{(N-N p-\lambda p N) / p}}{(N p(1+\lambda)-N)^{1 / p}} \\
& \cdot \frac{r^{\left(\lambda p^{\prime} N+N\right) / p^{\prime}}}{\left(\lambda p^{\prime} N+N\right)^{1 / p^{\prime}}}=\frac{(p-1)^{1 / p^{\prime}}}{p(1+\lambda)-1}<\infty
\end{aligned}
$$

Hence $I_{2} \leq \frac{p}{p(1+\lambda)-1}\left(\int_{\mathbb{R}^{N}} \frac{|u(x)|^{p}}{|x|^{p / N}} d x\right)^{1 / p}$ and inequality (6.1) follows by combining the last estimate with (6.2)-(6.3) and subtracting. 
Remark 6.1 Inequality (6.1) is an $N$-dimensional counterpart of inequality (1.5), i.e., with weight 1 on the right hand side. Of course, also more general cases can be considered. Let us mention at least the following more dimensional extension of Theorem 4.1; we omit the proof since the arguments are quite similar to the onedimensional case.

THEOREM 6.2 Let $\omega(x, y)$ be a non-negative measurable function on $\mathbb{R}^{N} \times$ $\mathbb{R}^{N}$, locally integrable in both variables separately. Let $1<p<\infty$ and $\lambda \geq-1 / p$ and denote $W(x)=\left(\frac{1}{|B(|x|)|} \int_{B(|x|)} \omega^{1-p^{\prime}}(x, t) d t\right)^{1-p}$. If

$$
C_{p}:=\sup _{r>0}\left\{\int_{|x| \geq r} \frac{W(x)}{|x|^{\lambda p N}} d x\right\}^{1 / p}\left\{\int_{|x| \leq r}|x|^{\lambda p^{\prime} N} W^{1-p^{\prime}}(x) d x\right\}^{1 / p^{\prime}}<\infty
$$

and $K=C_{p} \frac{p}{(p-1)^{1 / p^{p}}} \frac{N^{1 / p}}{\left|S^{N-1}\right|}<1$, then

$$
\begin{aligned}
& \left(\int_{\mathbb{R}^{N}} \frac{W(x)}{\left.|x|^{\lambda p N}|u(x)|^{p} d x\right)^{1 / p}}\right. \\
& \quad \leq \frac{1}{1-K} \frac{N^{1 / p} 2^{N(1+\lambda p) / p}}{\left|S^{N-1}\right|^{1 / p}}\left(\int_{\mathbb{R}^{N}} \int_{\mathbb{R}^{N}} \frac{|u(x)-u(y)|^{p}}{|x-y|^{N(1+\lambda p)}} \omega(x, y) d y d x\right)^{1 / p}
\end{aligned}
$$

\section{Acknowledgements}

The work of the second author was partially supported by the Grant Agency of Czech Republic, Grant No. 201/94/1066.

\section{References}

[1] V. Burenkov and W.D. Evans, Weighted Hardy's inequalities for differences and the extension problem for spaces with generalized smoothness (to appear).

[2] P. Grisvard, Espaces intermédiaires entre espaces de Sobolev avec poids, Ann. Scuola. Norm. Sup. Pisa., 23 (1969), 373-386.

[3] G.N. Jakovlev, Boundary properties of functions from the space $W_{p}^{(l)}$ on domains with angular points (Russian), Dokl. Akad. Nauk SSSR, 140 (1961), 73-76.

[4] A. Kufner and L.E. Persson, Hardy inequalities of fractional order via interpolation, WSSIAA (1994), 417-430.

[5] A. Kufner and H. Triebel, Generalizations of Hardy's inequality, Conf. Sem. Mat. Univ. Bari, 156 (1978), 21 pp. 
[6] L. Maligranda, Generalized Hardy inequalities in rearrangement invariant spaces, J. Math. pures et appl., 59 (1980), 405-415.

[7] B. Opic and A. Kufner, Hardy-type inequalities, Longman Scientific \& Technical, Harlow (1990).

[8] G. Palmieri, Un approcio alla teoria degli spazi di traccia relativi agli spazi di Orlicz-Sobolev, Bol. U.M.I., (5), 16-B (1979), 100-109.

[9] H. Triebel, Interpolation Theory, Function Spaces, Differential Operators. $2^{\text {nd }}$ edition, Johann Ambrosius Barth Verlag, Heidelberg-Leipzig, 1995. 